\title{
A multiple time step algorithm for trajectory surface hopping simulations
}

Pablo Baudin ${ }^{1, \text { a) }}$ and Ursula Rothlisberger ${ }^{1, b)}$

Laboratory of Computational Chemistry and Biochemistry, Institute of

Chemical Sciences and Engineering, École Polytechnique Fédérale de Lausanne, CH-1015 Lausanne, Switzerland

(Dated: 25 February 2021)

A multiple time step (MTS) algorithm for trajectory surface hopping molecular dynamics has been developed, implemented, and tested. The MTS scheme is an extension of the $a b$ initio implementation for Born-Oppenheimer molecular dynamics presented in [J. Chem. Theory Comput. 14, 2834 (2018)]. In particular, the MTS algorithm has been modified to enable the simulation of non-adiabatic processes with the trajectory surface hopping (TSH) method and Tully's fewest switches algorithm. The specificities of the implementation lie in the combination of Landau-Zener and Tully's transition probabilities during the inner MTS time steps. The new MTS-TSH method is applied successfully to the photorelaxation of protonated formaldimine, showing that the important characteristics of the process are recovered by the MTS algorithm. A computational speed-up between 1.5 and 3 has been obtained compared to standard TSH simulations which is close to the ideal values that could be obtained with the computational settings considered.

a) pablo.baudin@epfl.ch

b) ursula.roethlisberger@epfl.ch 


\section{CONTENTS}

I. Introduction $\quad 3$

II. Theory

A. Trajectory surface hopping in CPMD 4

1. Tully's fewest switches method 5

2. Landau-Zener transition probabilities 6

B. Trajectory surface hopping with multiple time step scheme 6

$\begin{array}{ll}\text { 1. Standard MTS algorithms } & 7\end{array}$

2. MTS algorithm for trajectory surface hopping dynamics 9

III. Results and Discussion 11

A. Investigating different approximations for the non-adiabatic couplings 11

B. Comparing single trajectories via deterministic surface hopping 14

1. Quality assessment 14

2. Efficiency assessment 16

$\begin{array}{ll}\text { C. Stochastic surface hopping } & 20\end{array}$

$\begin{array}{ll}\text { IV. Conclusions and outlook } & 24\end{array}$

Supplementary Material 25

$\begin{array}{ll}\text { Author information } & 25\end{array}$

$\begin{array}{ll}\text { Contributions } & 25\end{array}$

$\begin{array}{ll}\text { Funding } & 26\end{array}$

$\begin{array}{ll}\text { Notes } & 26\end{array}$

$\begin{array}{ll}\text { Acknowledgments } & 26\end{array}$

$\begin{array}{ll}\text { References } & 26\end{array}$ 


\section{INTRODUCTION}

Non-adiabatic phenomena such as photo-physical or photo-chemical processes are characterized by a failure of the Born-Oppenheimer (BO) approximation commonly invoked to describe molecular systems. The BO approximation, or the closely related adiabatic approximation, decouples the description of the nuclei and electrons. In non-adiabatic processes this coupling becomes important and the BO approximation breaks down.

Many different methods have been developed in order to describe non-adiabatic processes, ranging from fully quantum and formally exact models to mixed quantum/classical or semiclassical approaches (for a review see Refs. 1 and 2). Each method has its pros and cons, but in most cases it boils down to a compromise between computational efficiency and accuracy. In this work, we focus on one of the most popular methods, the trajectory surface hopping (TSH) approach.

In the TSH method (summarized in section II A), the evolution of the system is represented by a swarm of independent classical nuclear trajectories, which can hop from one electronic state to another in a stochastic way. The forces acting on the nuclei are calculated on-the-fly along each trajectory and transitions between electronic levels are considered simultaneously. In this way, the TSH method is thus able to describe non-adiabatic phenomena such as photo-chemical and photo-physical processes.

The TSH approach belongs to the mixed quantum/classical class of methods and is one of the computationally most expedient way to include non-adiabatic effects. Nonetheless, TSH simulations require the evaluation of the nuclear forces from first-principles simulations, i.e., by solving the time-independent electronic Schrödinger equation, and those forces have to be evaluated for each nuclear geometry along the trajectory. Furthermore, due to its stochastic form (see section II A), the TSH approach requires to run a statistical ensemble of trajectories, which means that, in practice, several hundred thousands of geometries have to be considered. ${ }^{2-4}$ Solving the electronic Schrödinger equation is very computationally demanding and often have a very steep scaling with the number of electrons considered. ${ }^{5,6}$ These considerations limit considerably the applications of the TSH method. In particular, the three main limitations arise from: (i) the size of the systems that can be treated, (ii) the number of trajectories required to recover the proper statistical properties, and (iii) the duration of the physical processes that can be studied, i.e. the total length of the simulations. 
In recent years, several attempts to extend the application range of non-adiabatic MD have been proposed. ${ }^{7-9}$ The present article is a further contribution to reduce the computational cost of non-adiabatic dynamics.

The strategy investigated in this work, is to reduce the computational requirements of the TSH method by relying on a multiple time step (MTS) algorithm. MTS techniques

have first been introduced by Tuckerman et al. in the context of classical MD. ${ }^{10}$ The MTS scheme relies on a decomposition of the atomic or nuclear forces into different components with different characteristic time scales. This decomposition enables to calculate the slow components of the forces less frequently than the fast one, while maintaining a fully timereversible symplectic propagation. If the computational cost of the slow components is significant, large computational speed-ups can thus be obtained.

This article is organized as follows. After introducing an MTS algorithm for TSH simulations in section II B, the new method is applied to the photorelaxation of protonated formaldimine (section III). The MTS-TSH algorithm is compared to standard TSH simulations both in terms of accuracy and computational cost. Finally, some concluding remarks and perspectives are given in section IV.

\section{THEORY}

In this first section, we briefly review the TSH formalism for non-adiabatic MD with particular emphasis on the version implemented in the CPMD plane-wave package. ${ }^{11}$

\section{A. Trajectory surface hopping in CPMD}

The TSH method can be seen as an attempt to introduce coupling between the electronic and nuclear degrees of freedom in Born-Oppenheimer molecular dynamics (BOMD). ${ }^{12}$ Standard BOMD consists in a description of the nuclear coordinates based on classical mechanics,

$$
M_{\alpha} \ddot{\mathbf{R}}_{\alpha}=\mathbf{F}_{\alpha}(\mathbf{R})
$$

where the index $\alpha$ denotes a given nucleus of mass $M_{\alpha}$ and classical coordinates $\mathbf{R}_{\alpha}$ and the two dots on top of the coordinates denote a second-order derivative with respect to time. When no index is specified, $\mathbf{R}$ stands for all nuclear coordinates. The forces acting 
on the nuclei, $\mathbf{F}_{\alpha}(\mathbf{R})$, are usually calculated on-the-fly, from ab initio electronic structure calculations at fixed nuclear geometries,

$$
\mathbf{F}_{\alpha}(\mathbf{R})=-\nabla_{\alpha} H_{L L}
$$

where the diagonal matrix elements of the molecular electronic Hamiltonian, $\hat{H}$ (under the BO approximation) are given by,

$$
H_{L L}=\left\langle\psi_{L}(\mathbf{r} ; \mathbf{R})|\hat{H}| \psi_{L}(\mathbf{r} ; \mathbf{R})\right\rangle
$$

In Eq. (3) we have introduced the electronic wavefunction, $\psi_{L}$, for an arbitrary adiabatic state, $L$, which depends on the electronic coordinates $\mathbf{r}$. The parametric dependence of the electronic wavefunction on the nuclear geometry, $\mathbf{R}$, is also given. Generally, a single BOMD trajectory will thus propagate the classical nuclear coordinates on a single PES corresponding to a specific adiabatic electronic state.

However, when more than one electronic state is important to describe the dynamics of a system (for example in the description of photo-physical phenomena) it is important to go beyond the BO approximation and consider non-adiabatic algorithms.

\section{Tully's fewest switches method}

One of the most popular approaches used to describe such phenomena is the TSH method, in particular when combined with Tully's fewest switches algorithm. ${ }^{2,13,14}$ In TSH a given trajectory can hop from one electronic state to another in a stochastic way, depending on the probability of the transition to occur. In order to get the transition probabilities one generally has to solve a time-dependent equation for the electrons of the system,

$$
i \hbar \dot{C}_{J}(t)=C_{J}(t) \omega_{J}-i \hbar \sum_{K}^{N_{\text {states }}} C_{K}(t) \sigma_{J K}(\mathbf{R})
$$

Where the time-dependent coefficients $C_{J}(t)$ comes from an expansion of the time-dependent

electronic wavefunction as a linear combination of time-independent adiabatic states. $N_{\text {states }}$ is the total number of electronic states considered, $\omega_{J}$ is the excitation energy for state $J$ and $\sigma_{J K}$ is the non-adiabatic coupling (NAC) term,

$$
\sigma_{J K}=\left\langle\psi_{J} \mid \partial_{t} \psi_{K}\right\rangle
$$


In CPMD, the NAC terms are computed by finite differences and using a CIS representation of the excited states. ${ }^{15-17}$ Finally, in the fewest switches (FS) scheme, the probability of transition from adiabatic states $J$ to $K$ is evaluated as,

$$
P_{J \rightarrow K}^{\mathrm{FS}}=-\frac{2 \cdot \delta t}{\left|C_{J}\right|^{2}} \cdot \mathfrak{R}\left(C_{K} C_{J}^{*} \sigma_{J K}\right)
$$

where $\delta t$ is the classical time step used to integrate eq. (1), $\mathfrak{R}(z)$ denotes the real part of $z$ and negative probabilities are set to zero. The decision to hop from state $J$ to state $K$ is then taken by generating a random number $r \in[0,1]$ and evaluating the following condition,

$$
\sum_{L=0}^{K-1} P_{J \rightarrow L}^{\mathrm{FS}}<r<\sum_{L=0}^{K} P_{J \rightarrow L}^{\mathrm{FS}} .
$$

\section{Landau-Zener transition probabilities}

As a simpler alternative to the solution of eq. (4) for the calculations of the transition probabilities in eq. (6), it is possible to obtain approximate transition probabilities from Landau-Zener-Stückelberg (LZ) theory for non-adiabatic transitions. ${ }^{18,19}$ In the CPMD package, such probabilities are computed directly from the knowledge of the energy of the adiabatic electronic states as,

$$
P_{J \rightarrow K}^{\mathrm{LZ}}=\exp \left(-\frac{\pi^{2}}{h} \cdot \frac{\left|\Delta E_{J K}^{\mathrm{adia}}\right|^{2}}{\max \left(\mathrm{d}\left|\Delta E_{J K}^{\text {adia }}\right| / \mathrm{d} t\right)}\right)
$$

where $\Delta E_{J K}^{\text {adia }}$ is the gap between adiabatic states $J$ and $K$ directly obtained as a byproduct of DFT and TDDFT calculations. ${ }^{18}$ In a TSH simulation relying on LZ theory, a hop from an electronic state to another is considered based on the following condition,

$$
P_{J \rightarrow K}^{\mathrm{LZ}}>r
$$

where $r$ is again a random number chosen between zero and one.

For more details about the implementation of TSH in the CPMD package, see Refs. 11,15 , and 20 .

\section{B. Trajectory surface hopping with multiple time step scheme}

MTS techniques have been introduced as a way to reduce the computational cost of molecular dynamics for systems in which the forces in action can be decomposed into different 
time scales. The success of MTS techniques is largely due to the development of the reversible reference system propagation algorithm (rRESPA) by Tuckerman et al. in Ref. 10.

Recently, some of us implemented rRESPA in the CPMD package for BOMD. ${ }^{21}$ The important details of this implementation are summarized in section IIB 1, while in section II B 2, we suggest an extension of the MTS algorithm to enable non-adiabatic dynamics in the context of TSH-MD.

\section{Standard MTS algorithms}

In Ref. 10, Tuckerman et al. introduced the Trotter factorization of the Liouville operator as a convenient way to generate reversible MD integrators. This technique is summarized here. Let us first consider a phase space element $\Gamma(t=0)$ which describes the initial positions $\left(x_{j}\right)$ and momenta $\left(p_{j}\right)$ of all the nuclei of a system. The phase space element can be propagated in time using a classical propagator, $G(t)$,

$$
\Gamma(t)=G(t) \Gamma(0)=e^{i L t} \Gamma(0)
$$

where $L$ is the Liouville operator given by,

$$
i L=\sum_{j}\left[\dot{x}_{j} \partial_{x_{j}}+F_{j} \partial_{p_{j}}\right]
$$

and $F_{j}$ is a single component of the nuclear forces (the index $j$ is a collective index for Cartesian coordinates and nuclei). By assuming a time scale separation of the forces into fast $\left(\mathbf{F}^{\text {fast }}\right)$, and slow $\left(\mathbf{F}^{\text {slow }}\right)$ components, it is possible to rewrite the Liouville operator as,

$$
\begin{gathered}
i L=i L_{x}+i L_{p}^{\text {fast }}+i L_{p}^{\text {slow }} \\
i L_{x}=\sum_{j} \dot{x}_{j} \partial_{x_{j}} \\
i L_{p}^{\text {fast }}=\sum_{j} F_{j}^{\text {fast }} \partial_{p_{j}} \\
i L_{p}^{\text {slow }}=\sum_{j} F_{j}^{\text {slow }} \partial_{p_{j}}
\end{gathered}
$$

Applying a Trotter factorization on the classical propagator and discarding terms of third order and higher in $t$, we can define a discrete time propagator, which can be translated into 
an MTS algorithm, ${ }^{10,22}$

$$
G^{\mathrm{MTS}}(\Delta t)=e^{i L_{p}^{\text {slow }}(\Delta t / 2)}\left[G^{\text {fast }}(\Delta t / N)\right]^{N} e^{i L_{p}^{\text {slow }}(\Delta t / 2)}
$$

with

$$
G^{\text {fast }}(\Delta t / N)=G^{\text {fast }}(\delta t)=e^{i L_{p}^{\text {fast }}(\delta t / 2)} e^{i L_{x} \delta t} e^{i L_{p}^{\text {fast }}(\delta t / 2)} .
$$

In eqs. (16) and (17) we have introduced two finite time steps; $\Delta t$, which reflects the time scale of the slow forces $\left(\mathbf{F}^{\text {slow }}\right)$, and $\delta t=\Delta t / N$, which is adapted to the fast forces $\left(\mathbf{F}^{\text {fast }}\right)$. It should now be apparent that such an MTS algorithm can lead to computational savings, if the slow forces that have to be calculated less frequently are computationally more demanding.

Algorithm 1 represents a pseudo-code that can be obtained by applying each term of the propagator in eq. (16) (one by one from the right to the left) onto an initial phase space element $\Gamma(0) \equiv\{\mathbf{x}, \mathbf{p}\}$.

1: Initialize positions, velocities, fast and slow forces: $\mathbf{x}, \mathbf{v}, \mathbf{F}^{\text {fast }}, \mathbf{F}^{\text {slow }}$

2: for $i=1$, maxiter do (MD loop for the slow component)

3: $\quad$ Slow velocity update: $\mathbf{v} \leftarrow \mathbf{v}+\frac{\Delta t}{2 m} \cdot \mathbf{F}^{\text {slow }}$

4: $\quad$ for $j=1, N$ do (MD loop for the fast component)

5: $\quad$ Fast velocity update: $\mathbf{v} \leftarrow \mathbf{v}+\frac{\delta t}{2 m} \cdot \mathbf{F}^{\text {fast }}$

6: $\quad$ Position update: $\mathbf{x} \leftarrow \mathbf{x}+\mathbf{v} \cdot \delta t$

7: $\quad$ Get fast components of the forces: $\mathbf{F}^{\text {fast }}$

8: $\quad$ Final fast velocity update: $\mathbf{v} \leftarrow \mathbf{v}+\frac{\delta t}{2 m} \cdot \mathbf{F}^{\text {fast }}$

9: $\quad$ end for (MD loop for the fast component)

10: $\quad$ Get slow components of the forces: $\mathbf{F}^{\text {slow }}$

11: $\quad$ Final slow velocity update: $\mathbf{v} \leftarrow \mathbf{v}+\frac{\Delta t}{2 m} \cdot \mathbf{F}^{\text {fast }}$

12: end for (MD loop for the slow component)

ALG. 1: Standard rRESPA MTS algorithm obtained as a direct translation of the discrete propagator in eq. (16). For comparison with the velocity Verlet algorithm, the steps concerned with momenta, $\mathbf{p}$, have been re-written in terms of velocities, $\mathbf{v}$.

In the context of first-principles BOMD, the separation of the forces into fast and slow components is not straightforward. In the CPMD package we have chosen to use different 
levels of electronic structure theory to decompose the forces. Typically, a "low" level density functional (e.g. GGA) is used to calculate the fast components of the nuclear forces, while the slow components are obtained as the difference between the forces obtained with a "higher" level functional (e.g. hybrid) and the "low" level forces,

$$
\begin{aligned}
\mathbf{F}^{\text {fast }} & =\mathbf{F}^{\text {low }} \\
\mathbf{F}^{\text {slow }} & =\mathbf{F}^{\text {high }}-\mathbf{F}^{\text {low }} .
\end{aligned}
$$

The physical motivation for this separation comes from the fact that the chosen electronic structure levels differ only in their treatment of correlation (or exchange and correlation in the case of DFT). Since those contributions correspond to relatively weak interactions in terms of energy (with no explicit dependence on nuclear positions) they can be expected to represent relatively weak/slowly varying force contributions.

The implementation of the MTS algorithm in CPMD makes use of this separation as well as a slightly different but completely equivalent layout of the code. ${ }^{21}$ This implementation presented in pseudo-code in algorithm 2, makes the MTS algorithm look like a velocity Verlet algorithm with effective forces, $\mathbf{F}^{\mathrm{eff}}$, that are time step dependent.

Even though, the force decomposition in terms of high and low electronic structure levels is done ad hoc, this kind of separation has already proven useful ${ }^{21,23}$ and the benefits in terms of computational cost are evident.

\section{MTS algorithm for trajectory surface hopping dynamics}

When using the MTS implementation described in algorithm 2 in combination with a TSH algorithm, one has to decide how to hop from one electronic state to another. In order to get trajectories of high accuracy, it would be beneficial to consider electronic transitions based on Tully's FS criterion in eq. (7) calculated with the MTS high level functional. In the following, this type of calculation (with a velocity Verlet algorithm) will actually be used as a reference. However, when using an MTS algorithm, if the outer time step $\Delta t$ becomes large, some parts of the PES where the transition probabilities are high might be treated only by the low level functional and the transitions would be missed at the high level.

In this work, we propose another strategy that can potentially solve that problem. This strategy consists in evaluating the transitions probabilities with the LZ formula in eq. (8) 
1: Initialize positions, velocities, high and low level forces: $\mathbf{x}, \mathbf{v}, \mathbf{F}^{\text {high }}, \mathbf{F}^{\text {low }}$

2: Get effective force: $\mathbf{F}^{\mathrm{eff}} \leftarrow \mathbf{F}^{\text {low }}+\left(\mathbf{F}^{\text {high }}-\mathbf{F}^{\text {low }}\right) \cdot N$

3: for $i=1$, maxiter do (MD loop)

4: $\quad$ Velocity update: $\mathbf{v} \leftarrow \mathbf{v}+\frac{\delta t}{2 m} \cdot \mathbf{F}^{\mathrm{eff}}$

5: $\quad$ Position update: $\mathbf{x} \leftarrow \mathbf{x}+\mathbf{v} \cdot \delta t$

6: $\quad$ if $i \equiv 0(\bmod N)$ then (outer step)

7: $\quad$ Get both high and low level forces, $\mathbf{F}^{\text {high }}, \mathbf{F}^{\text {low }}$

8: $\quad$ Get effective force: $\mathbf{F}^{\mathrm{eff}} \leftarrow \mathbf{F}^{\text {low }}+\left(\mathbf{F}^{\text {high }}-\mathbf{F}^{\text {low }}\right) \cdot N$

9: $\quad$ else (inner step)

10: $\quad$ Effective forces are set to the low level forces: $\mathbf{F}^{\mathrm{eff}} \leftarrow \mathbf{F}^{\text {low }}$

11: $\quad$ end if (outer/inner steps)

12: $\quad$ Final velocity update: $\mathbf{v} \leftarrow \mathbf{v}+\frac{\delta t}{2 m} \cdot \mathbf{F}^{\mathrm{eff}}$

13: end for (MD loop)

ALG. 2: MTS-BOMD algorithm as implemented in the CPMD package. This algorithm can be obtained straightforwardly from algorithm 1 by using the partitioning of the forces in eqs. (18) and (19) and reshuffling a few steps.

during the low level steps, while for high level steps, the electronic transition are evaluated according to the FS criterion in eq. (7) based on the high level quantities.

This is not yet completely satisfactory since it does not guarantee that a transition detected with the LZ probabilities during a low level step would have also been detected by the FS criterion calculated with the high level functional. To further improve on that issue, we suggest that if a transition is detected at the low level (using LZ theory), a high level calculation is triggered to confirm the transition using Tully's FS criterion. This strategy is described in algorithm 3 and tested in the remaining sections.

Finally, it is important to note that in the case of high level calculations triggered by the low level LZ criterion, the random number used in the high level FS criterion in eq. (7), should be the same as in the low level LZ criterion in eq. (9). We also underline that the discrete time step $\delta t$ in eq. (6) always correspond to the inner time step in the MTS scheme. This can be rationalized by realizing that at each inner time step, an electronic transition can occur if it is detected at the low level and confirmed at the high level, such that when 
eq. (6) is invoked, it is only to check for a transition in the last $\delta t$ time window.

\section{RESULTS AND DISCUSSION}

In this section, the protonated formaldimine $\left(\mathrm{CH}_{2} \mathrm{NH}_{2}^{+}\right.$, denoted as system I) is used as a simple yet interesting example to investigate the capabilities of the new MTS-TSH method presented in section II B. In particular, we will investigate the possibility to use the MTS-TSH algorithm as a more efficient alternative to the standard FS-TSH algorithm. The

physical process under investigation in this section is the photorelaxation of system I. This process is a typical example of photo-dynamics and is thus very handy to test new models for non-adiabatic molecular dynamics. $9,15,24-26$

All the calculations presented in this section have been performed with a local version of the CPMD plane-wave package. ${ }^{11}$ For the reference TSH simulations, the nuclear forces and NACs are computed with the PBE0 hybrid functional. ${ }^{27}$ The same functional is thus used for the high level forces in the MTS calculations, while the low level forces are obtained from the PBE functional. ${ }^{28,29}$ For a fair comparison, all remaining parameters are kept the same in the reference and the MTS simulations.

The five lowest singlet excited states obtained with the Tamm-Dancoff approximation of TDDFT are considered for all calculations. Norm-conserving Trouiller-Martins pseudopotentials are used with a plane-wave cutoff of $70 \mathrm{Ry}$ and an isolated cubic box with an edge of $10 \AA$. Unless specified otherwise, the inner time step is set to $\delta t=10$ a.u. See the supplementary materials for the raw data, the analysis scripts, and a full description of the computational details.

\section{A. Investigating different approximations for the non-adiabatic couplings}

First of all, it is important to rationalize the use of the LZ transition probabilities at the low level in the MTS-TSH method presented in section II B. For that purpose, we have run a reference trajectory for about 100 femtoseconds (fs) on the second excited state of system I. This trajectory was performed with the PBE0 functional and a standard velocity Verlet algorithm. The transitions probabilities where calculated at each time step using the FS method (see section II A 1) but no electronic transitions were allowed such that the system 
1: Initialize positions, velocities, and running electronic state: $\mathbf{x}, \mathbf{v}, J$

2: Initialize high and low level forces: $\mathbf{F}^{\text {high }}, \mathbf{F}^{\text {low }}$

3: Get effective force: $\mathbf{F}^{\mathrm{eff}} \leftarrow \mathbf{F}^{\text {low }}+\left(\mathbf{F}^{\text {high }}-\mathbf{F}^{\text {low }}\right) \cdot N$

4: for $i=1$, maxiter do (MD loop)

5: $\quad$ Velocity update: $\mathbf{v} \leftarrow \mathbf{v}+\frac{\delta t}{2 m} \cdot \mathbf{F}^{\mathrm{eff}}$

6: $\quad$ Position update: $\mathbf{x} \leftarrow \mathbf{x}+\mathbf{v} \cdot \delta t$

7: $\quad$ if $i \equiv 0(\bmod N)$ then (outer step)

8: $\quad$ Get both high and low level forces, $\mathbf{F}^{\text {high }}, \mathbf{F}^{\text {low }}$

9: $\quad$ Get effective force: $\mathbf{F}^{\mathrm{eff}} \leftarrow \mathbf{F}^{\text {low }}+\left(\mathbf{F}^{\text {high }}-\mathbf{F}^{\text {low }}\right) \cdot N$

10: $\quad$ Get high level FS transition probabilities: $P_{J \rightarrow K}^{\mathrm{FS}}$

11: $\quad$ if FS criterion in eq. (7) is met for any state $K \neq J$ then

12: $\quad$ Update index of adiabatic electronic state: $J \leftarrow K$

13: $\quad$ end if

14: $\quad$ else (inner step)

15: $\quad$ Effective forces are set to the low level forces: $\mathbf{F}^{\mathrm{eff}} \leftarrow \mathbf{F}^{\text {low }}$

16: $\quad$ Get low level LZ transition probabilities: $P_{J \rightarrow K}^{\mathrm{LZ}}$

17: $\quad$ if LZ criterion in eq. (9) is met for any state $K \neq J$ then (check at high level)

18: $\quad$ Effective forces are now set to the high level forces: $\mathbf{F}^{\mathrm{eff}} \leftarrow \mathbf{F}^{\text {high }}$

19: $\quad$ Get high level FS transition probabilities: $P_{J \rightarrow K}^{\mathrm{FS}}$

20: $\quad$ if FS criterion in eq. (7) is met for any state $K \neq J$ then

21: $\quad$ Update index of adiabatic electronic state: $J \leftarrow K$

22: $\quad$ end if

23: $\quad$ end if

24: $\quad$ end if (outer/inner steps)

25: $\quad$ Final velocity update: $\mathbf{v} \leftarrow \mathbf{v}+\frac{\delta t}{2 m} \cdot \mathbf{F}^{\mathrm{eff}}$

26: end for (MD loop)

ALG. 3: MTS-TSH algorithm as implemented in the CPMD package. This algorithm corresponds to a modified version of algorithm 2 that accounts for non-adiabatic transitions. See section II B for details. 


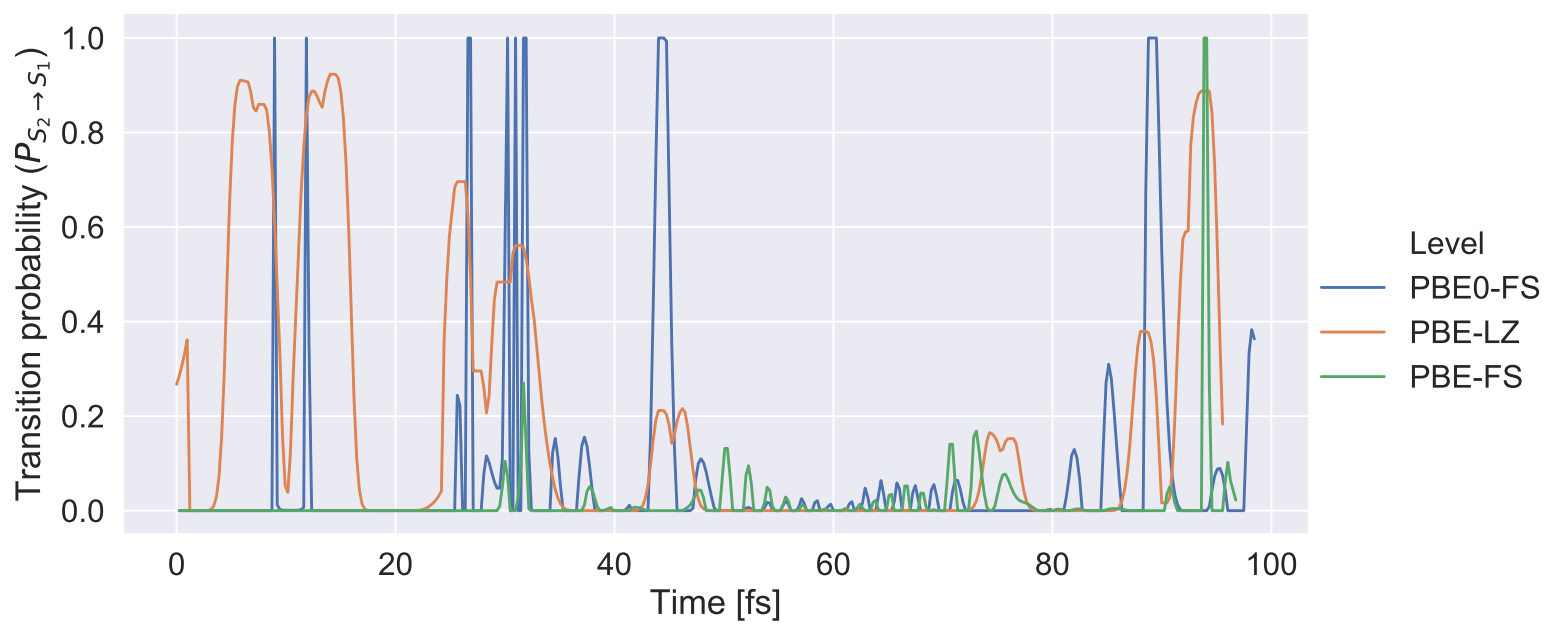

FIG. 1: Evolution of the transition probabilities (from $\mathrm{S}_{2}$ to $\mathrm{S}_{1}$ ) along a single trajectory started in the second excited state of system I. Three different levels are compared; PBE0-FS, corresponding to eq. (6) together with the PBE0 functional, PBE-LZ, corresponding to eq. (8) together with the PBE functional, and PBE-FS, corresponding to eq. (6) together with the PBE functional. (Values of $P_{S_{2} \rightarrow S_{1}}^{\mathrm{FS}}$ larger than 1, have been set back to unity.)

stayed on the second excited states PES the whole time.

The exact same trajectory (velocities and coordinates) have then been repeated by calculating the transitions probabilities using the LZ method (see section II A 2) together with the PBE functional (low level). For comparison, an additional run was performed at the PBE level and calculating transition probabilities using the FS method. No MTS algorithm was used in this section and the only difference between the three trajectories is the model used to calculate the transition probabilities (velocities and coordinates are the same for all three trajectories). Such conditions allows to compare the transition probabilities obtained with three different levels: PBE0-FS, PBE-LZ, and PBE-FS.

In Fig. 1 we have represented the evolution of the transition probabilities $\left(P_{S_{2} \rightarrow S_{1}}\right)$ for the three different levels. From Fig. 1, it is clear that neither the PBE-LZ probabilities nor the PBE-FS probabilities represent a completely reliable approximation to the reference PBE0-FS transition probabilities. However, a relatively good correlation is observed. In particular, whenever the reference PBE0-FS transition probabilities are large, the PBE-LZ probabilities are also relatively large. For some reason this is less obvious for the PBE-FS curve. 
The algorithm developed in section II B relies on the low level calculations to detect potential electronic transitions. Whenever a low level transition is detected, it is double checked with a high level FS calculation such that the low level probabilities do not have to be quantitatively accurate. The transition probabilities in Fig. 1 indicates that using LZ theory at the low level for the calculation of the transition probabilities is enough. In other words, the LZ probabilities can be used as a proxy during the low level steps of the MTS-TSH method.

We note that the objective of the investigation performed in this section is to support the design of the algorithm presented in section II B and that further tests could be performed to draw more general conclusions. Nonetheless, since the usage of LZ probabilities at the low level is only used to trigger high level calculations, we believe that a strong empirical support is not required at this stage.

\section{B. Comparing single trajectories via deterministic surface hopping}

As we have seen in section II A, non-adiabatic dynamics performed with a TSH algorithm are stochastic by nature due to the randomness used in the hopping procedure. This stochastic behaviour makes it difficult to compare individual trajectories obtained with a TSH algorithm. To properly compare different non-adiabatic models, one needs to look at a statistical ensemble of trajectories. Before we present such results in section IIIC, we first consider in this section a deterministic version of TSH in which the random number $r$ used in eqs. (7) and (9) has been fixed arbitrarily to $r=0.3$. We note that, the only MTS algorithm tested here and in the next section is the one presented in section II B, i.e., low level (PBE) LZ transition probabilities are used during the inner steps to trigger a high level (PBE0) calculation which confirms or not the electronic transition.

\section{Quality assessment}

Six different runs have been produced, all starting in the second excited state and with the same nuclear configuration. For simplicity, the nuclear velocities are initialized to zero. The first run is a reference TSH trajectory at the PBE0 level, while the 5 remaining trajectories are obtained with the MTS-TSH algorithm, and an MTS time step factor of 


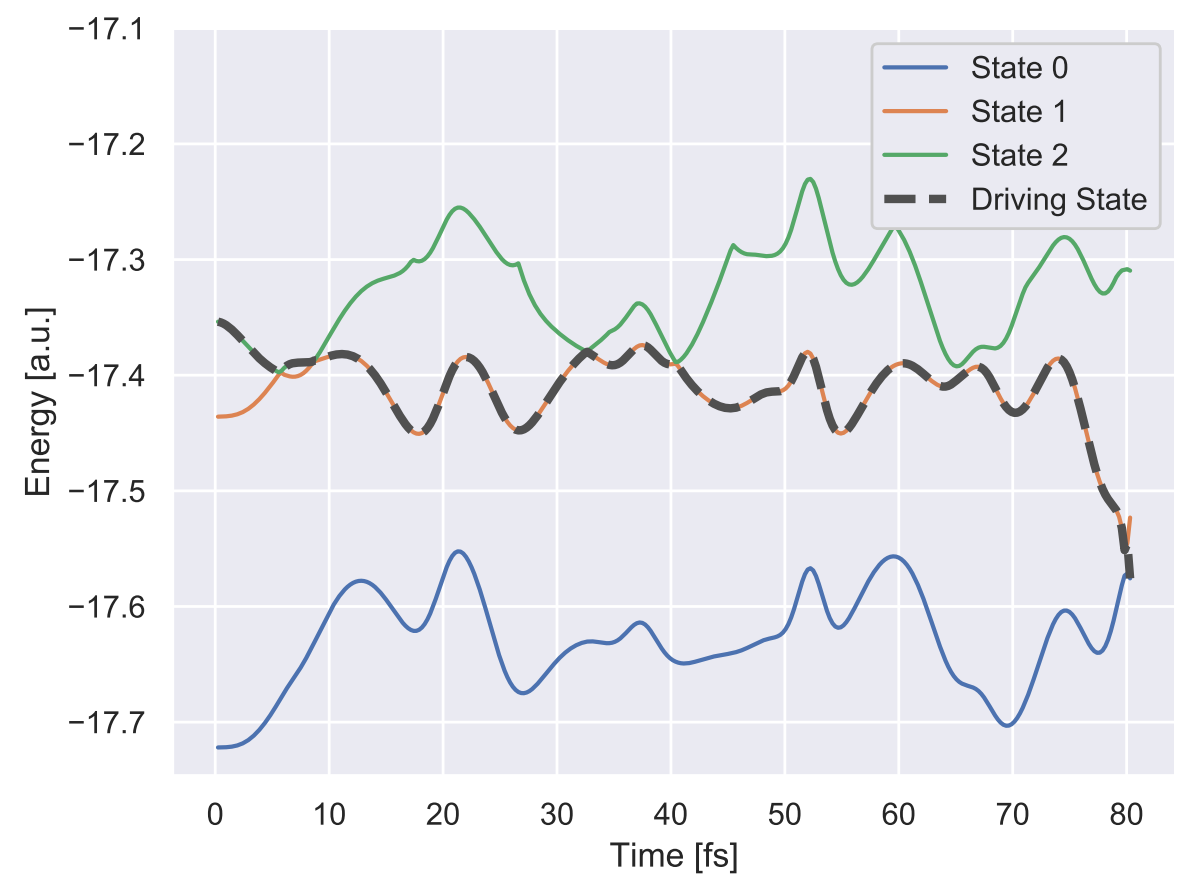

FIG. 2: Potential energy surfaces of the three lowest singlet states of system I obtained from an 80 femtoseconds PBE0 FS-TSH run started in the second excited state.

$N=\{2,3,4,6,8\}$.

In Fig. 2, the potential energy of the three lowest singlet states obtained from the reference PBE0 trajectory is represented. The trajectory starts in the second excited state and hops to the first excited state in less than $10 \mathrm{fs}$. The system stays in the first excited state for the next $70 \mathrm{fs}$ until it intersects with and hops into the ground state. All trajectories are stopped whenever they collapse into the ground state.

In Fig. 3, the potential energies of all runs (reference and MTS) are represented. For the MTS runs, we only plot the energy from the PBE0 steps, which explains why for the larger MTS factors, the curves appear less smooth. One can see that all the MTS trajectories, except with MTS factor 8, successfully describe the first transition from $\mathrm{S}_{2}$ to $\mathrm{S}_{1}$ in the first $10 \mathrm{fs}$. Between 30 and $40 \mathrm{fs}$, the reference trajectory enters a new non-adiabatic region as the first and second excited states become close in energy for the third time. Until that point, the MTS trajectories with MTS factor 2,3, 4, and 6 seem to describe the reference trajectory relatively well. Afterwards, the MTS trajectories with factor 2, 3, and 4 start to diverge from the reference run. The MTS trajectory with factor 6 is overall the closest from 
the reference run. However, an MTS factor of 8 seems to be too large to reproduce most of the features of the reference run. This analysis is confirmed by looking at the root-meanssquare-deviation (RMSD) of the nuclear positions of the MTS trajectories with respect to the reference run in Fig. 4.

The results presented in Figs. 3 and 4, could be interpreted as rather discouraging. Indeed, with only an MTS factor of 2, the MTS trajectory and the reference one start to diverge only after 35 fs. However, unlike with ground state MD, the presence of non-adiabatic events has a drastic impact on the chaotic behaviour of excited state dynamics. Very tiny differences in the nuclear positions and velocities or wavefunction parameters can lead to completely different trajectories. The fact that some of the MTS trajectories presented in Fig. 3 differ significantly from the reference trajectory does not mean that those trajectories are not physical. Only a statistical analysis of the photorelaxation process can enlighten us on that matter.

The deterministic investigation of the MTS-TSH implementation presented here indicates that it is possible to recover the main characteristics of a reference calculation using the MTS-TSH algorithm presented in section II B, for example in the case of MTS factor 6 . In section III C, we will investigate the possibility to recover statistically relevant quantities from the MTS-TSH algorithm, while in the next section we analyze the speed-ups obtained in the deterministic MTS-TSH simulations.

\section{Efficiency assessment}

Let us call $t^{\mathrm{FS}}=t^{\text {high }}$ the average CPU time per step spent in a standard FS-TSH simulation with a "high" level functional. This time takes into account the SCF optimization, the solution of the TDDFT equations as well as the calculation of the FS probabilities. In the following we use $t^{\mathrm{FS}}$ as a reference CPU time. In order to provide fair comparisons, we also need to consider the average CPU time per step from a LZ-TSH simulation with a "low" level functional $t^{\mathrm{LZ}}=t^{\text {low }}$.

The expected or ideal averaged CPU time per step in the MTS-TSH algorithm can then be calculated as,

$$
t^{\mathrm{MTS}(\mathrm{N})}=t^{\text {low }}+\frac{1}{N} \cdot t^{\text {high }}+X \cdot t^{\text {high }},
$$




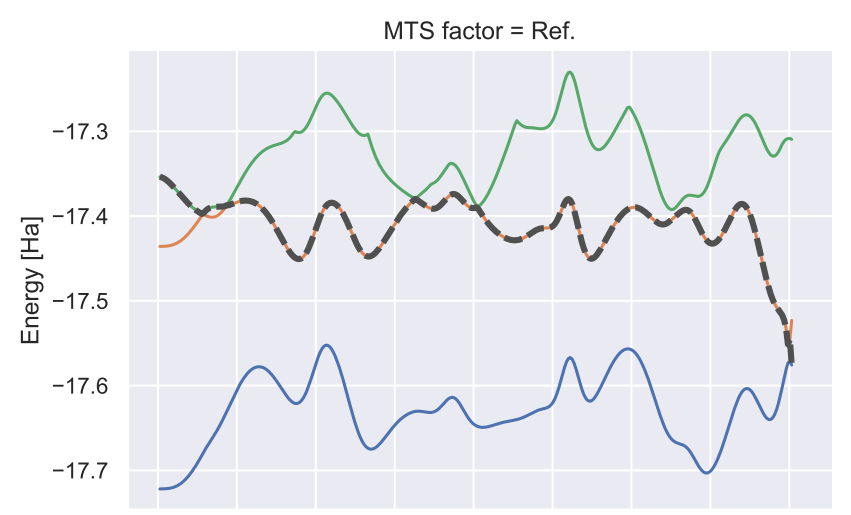

MTS factor $=3$

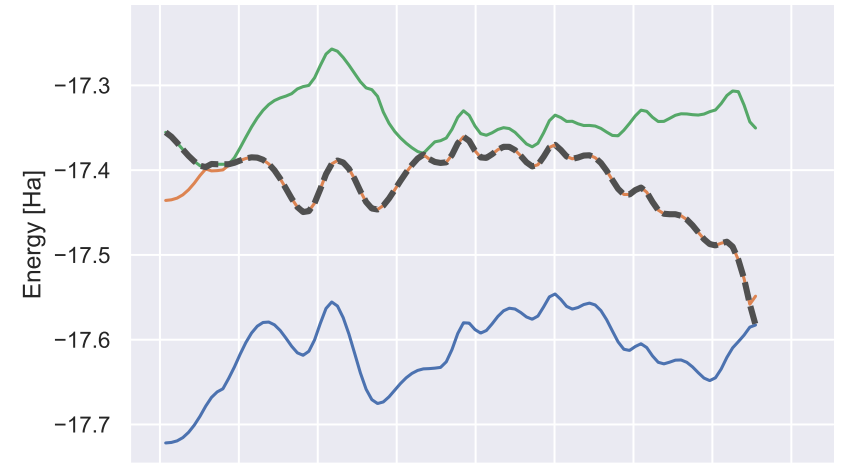

MTS factor $=6$

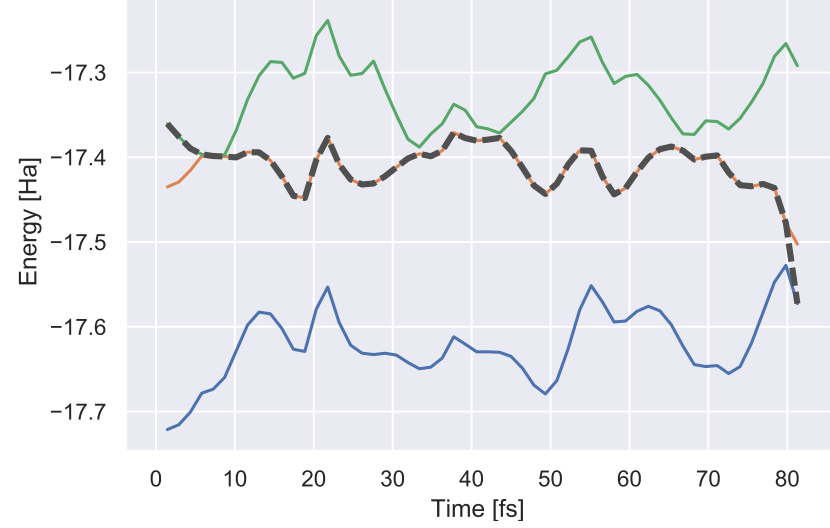

MTS factor $=2$

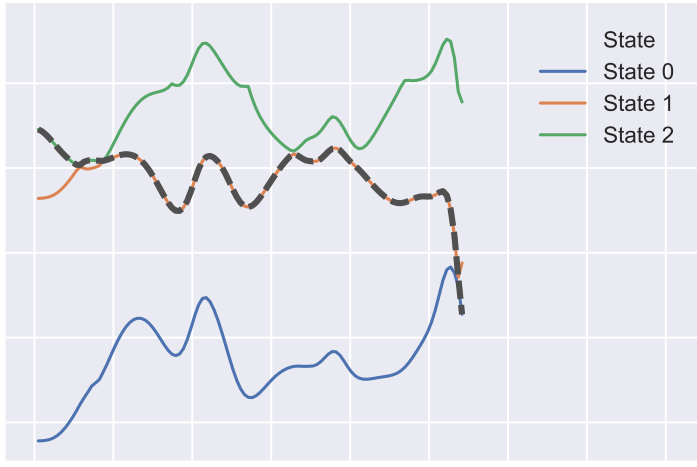

MTS factor $=4$

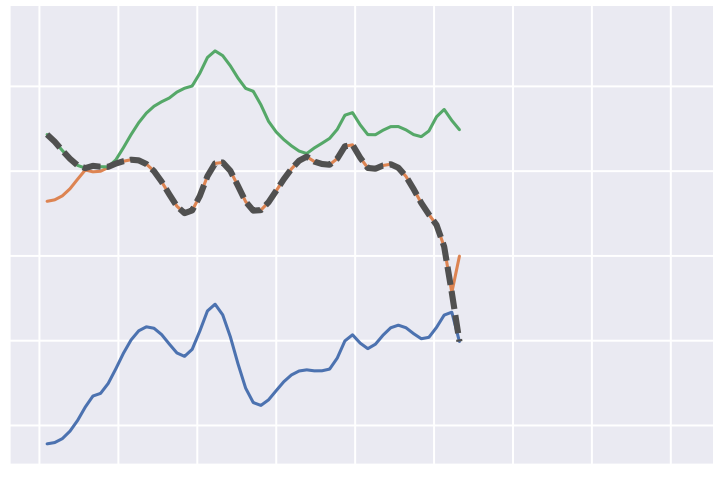

MTS factor $=8$

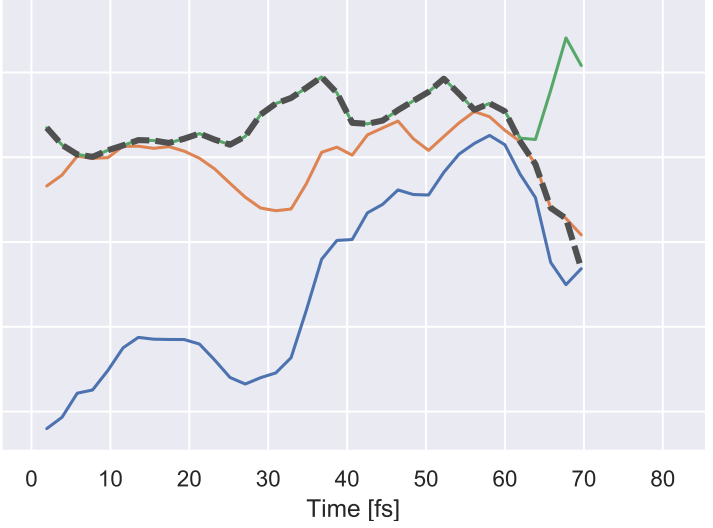

FIG. 3: Potential energy surfaces of the three lowest singlet states of system I obtained from 6 different calculations. The upper-left panel constitutes the reference PBE0 FS-TSH run, while the other panels represent the MTS-TSH runs with different MTS factors $(N=\{2,3,4,6,8\})$. All simulations have been started from the same geometries and zero velocities. The driving state is represented with the dashed thick black line.

where $N$ is the MTS factor and, $t^{\text {high }}$ and $t^{\text {low }}$ are CPU timings coming from standard (non MTS) simulations. $X$ denotes the average frequency of triggered high level steps which is a quantity difficult to predict. To obtain the ideal MTS-TSH speed-up we set $X=0$, and 


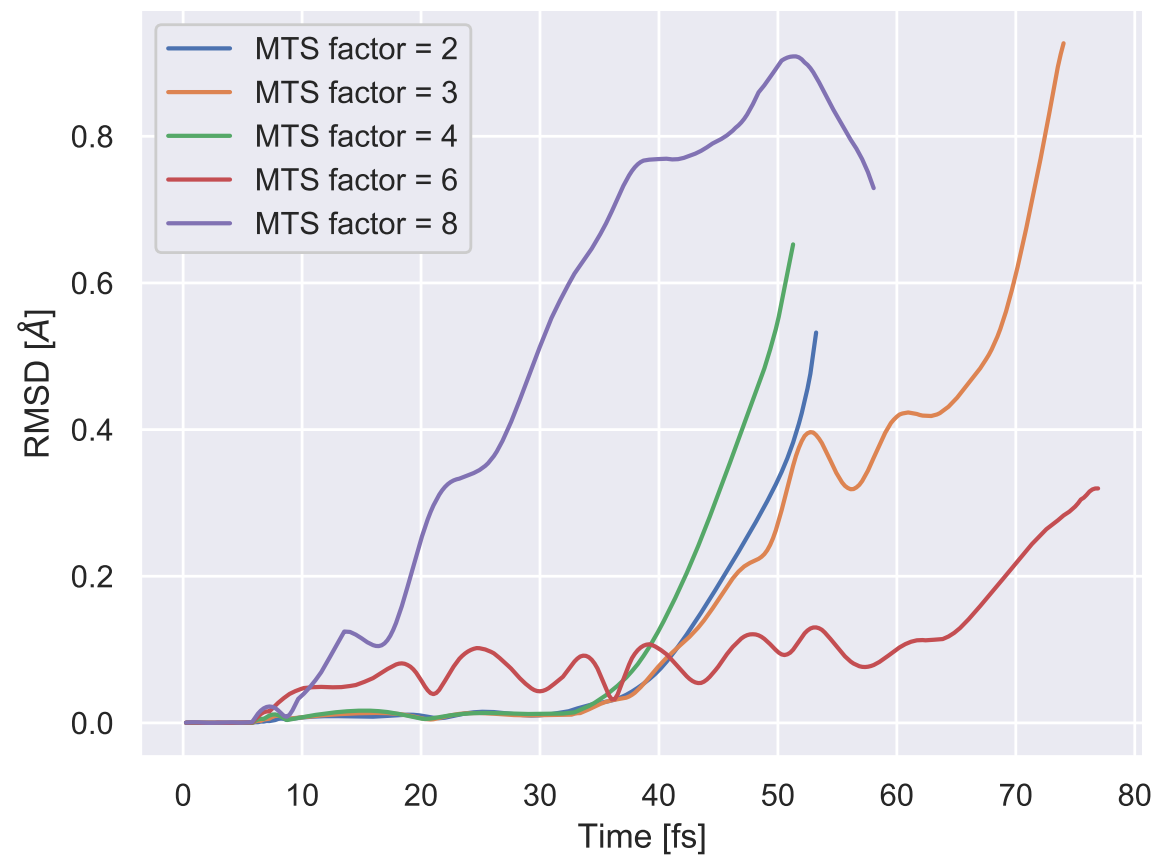

FIG. 4: Root-means-square-deviations (RMSD) of nuclear positions of the MTS trajectories with different MTS factors $(N=\{2,3,4,6,8\})$ with respect to a reference PBE0 FS-TSH trajectory. get,

$$
S^{\text {ideal }}=\frac{t^{\mathrm{FS}}}{t^{\mathrm{MTS}(\mathrm{N})}}=\frac{t^{\text {high }}}{t^{\text {low }}+\frac{1}{N} \cdot t^{\text {high }}}=\frac{N}{N \cdot \frac{t^{\text {low }}}{t^{\text {high }}}+1}
$$

In the limit of a negligible cost of the low level steps (compared to the high level ones) we get,

$$
S^{\text {limit }}=\lim _{t^{\text {high }} \gg t^{\text {low }}} S^{\text {ideal }}=N
$$

In practice, several things can impact the ideal and limit speed-ups. The most obvious one being the number of triggered high level steps. It is easy to realize that for large values of $N$, the number of triggered high level steps will tend towards a system dependent number, in most cases larger than zero. Such that one cannot achieve arbitrarily large speed-ups simply by increasing $N$. From a more practical point of view, the physics of the system (vibrational frequencies) will be the first parameter to consider as a limitation for the value of $N$ and thus for the speed-up (too large values of $N$ would lead to artifacts such as resonances ${ }^{30}$ ). A less straightforward impact on the effective (or real) speed-up is given by the overhead due 

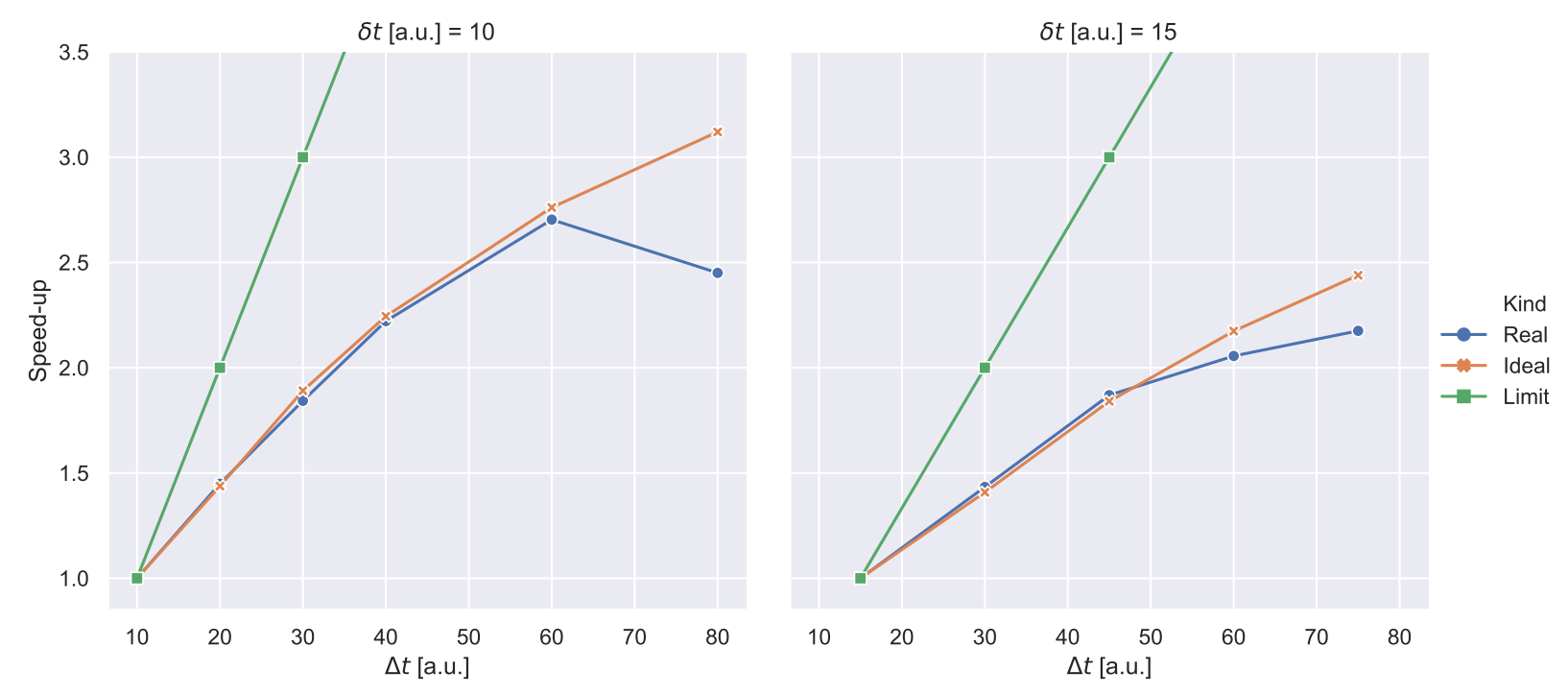

FIG. 5: Real MTS speed-up obtained from deterministic MTS-TSH calculations with different inner and outer time steps ( $\delta t$ and $\Delta t$, respectively) compared to reference PBE0 FS-TSH timings. For comparison the ideal and limit speed-ups as defined in eqs. (21) and (22), respectively, are also reported.

to the convergence of the high level (TD)DFT parameters. Indeed, since with increasing values of $N$, larger nuclear displacements occur between high level steps, the initial guess for the electronic structure calculation becomes less appropriate, often resulting in more (e.g. $\mathrm{SCF}$ ) iterations and thus higher computational requirements.

In Fig. 5, we have represented the ideal and limit MTS speed-ups as calculated from eqs. (21) and (22), respectively, as well as the real MTS speed-up obtained for the calculations presented in section III B (system I with the PBE0 and PBE functionals). The left panel of Fig. 5 corresponds to the MTS calculations with $\delta t=10$ a.u. and MTS factors $N=$ $\{2,3,4,6,8\}$, while, in the right panel a new set of calculations with $\delta t=15$ a.u. and MTS factors $N=\{2,3,4,5\}$ (all other parameters unchanged) are represented. The first points of both plots (with speed-up one) correspond to the reference FS-TSH calculations. The real MTS speed-ups are simply obtained from the ratio between $t^{\text {FS-PBE0 }}$ and the averaged time per step in the actual MTS simulations,

$$
S^{\text {real }}=\frac{t^{\text {MTS-total }}}{N^{\text {steps }}}
$$

From Fig. 5, we can see that both the real and ideal speed-ups are quite far from the 
limit speed-ups (up to a factor 3 of difference). This is simply a consequence of the fact that the condition, $t^{\text {high }} \gg t^{\text {low }}$ is not satisfied here. For $\delta t=10$ a.u. $t^{\text {high }} \simeq 5.1 \cdot t^{\text {low }}$, while for $\delta t=15$ a.u. $t^{\text {high }} \simeq 4.8 \cdot t^{\text {low }}$. It means that one way of improving the efficiency of MTS techniques is to consider cheaper "low" level models.

The real and ideal speed-ups are much closer from each-other and with an MTS factor between 3 and 6 it seems that reliable results could be obtained with a speed-up factor ranging from 1.5 to 2.5. The number of triggered high level calculations does not seem to affect the speed-up significantly since, in the considered calculations, the maximum averaged frequency of triggered high level calculations $[X$ in eq. (20)] is equal to 0.026, which corresponds to a triggered call every 38 inner step. Most of the differences between the real and ideal speed-up in Fig. 5 can thus be attributed to the convergence overhead discussed above.

However, strong variations are observed for the individual time per step (not averaged) along the trajectories. Surprisingly, the average time per step in the low level steps of the MTS runs are often lower than in the standard low level runs $\left(t^{\mathrm{LZ}-\mathrm{PBE}}\right)$. This explains why in Fig. 5 the ideal speed-up is sometimes lower than the real speed-up.

The different timings reported in this section are clearly subject to strong variations depending on the system considered as well as the computational parameters and the nuclear geometries. Therefore, a reliable comparison of the efficiency of the methods under investigation is a difficult task that will be further pursued in section III C.

\section{Stochastic surface hopping}

Starting from a PBE BOMD trajectory of 24 ps at $300 \mathrm{~K}$ in the ground state of system I, we have selected 100 equally spaced configurations. For each configuration, we have calculated the 5 lowest singlet excitation energies and oscillator strengths at the PBE0/TDDFT level. The starting state for the non-adiabatic dynamics is decided by randomly picking among the five lowest excited states with a distribution given by the normalized oscillator strengths at the corresponding configuration. From this random distribution, 94 runs started in the second excited state, one in the third, and five in the fourth excited state. The starting atomic positions and velocities were taken from the ground state MD. All the details concerning the preparation of the non-adiabatic simulations can be found in the SI. 


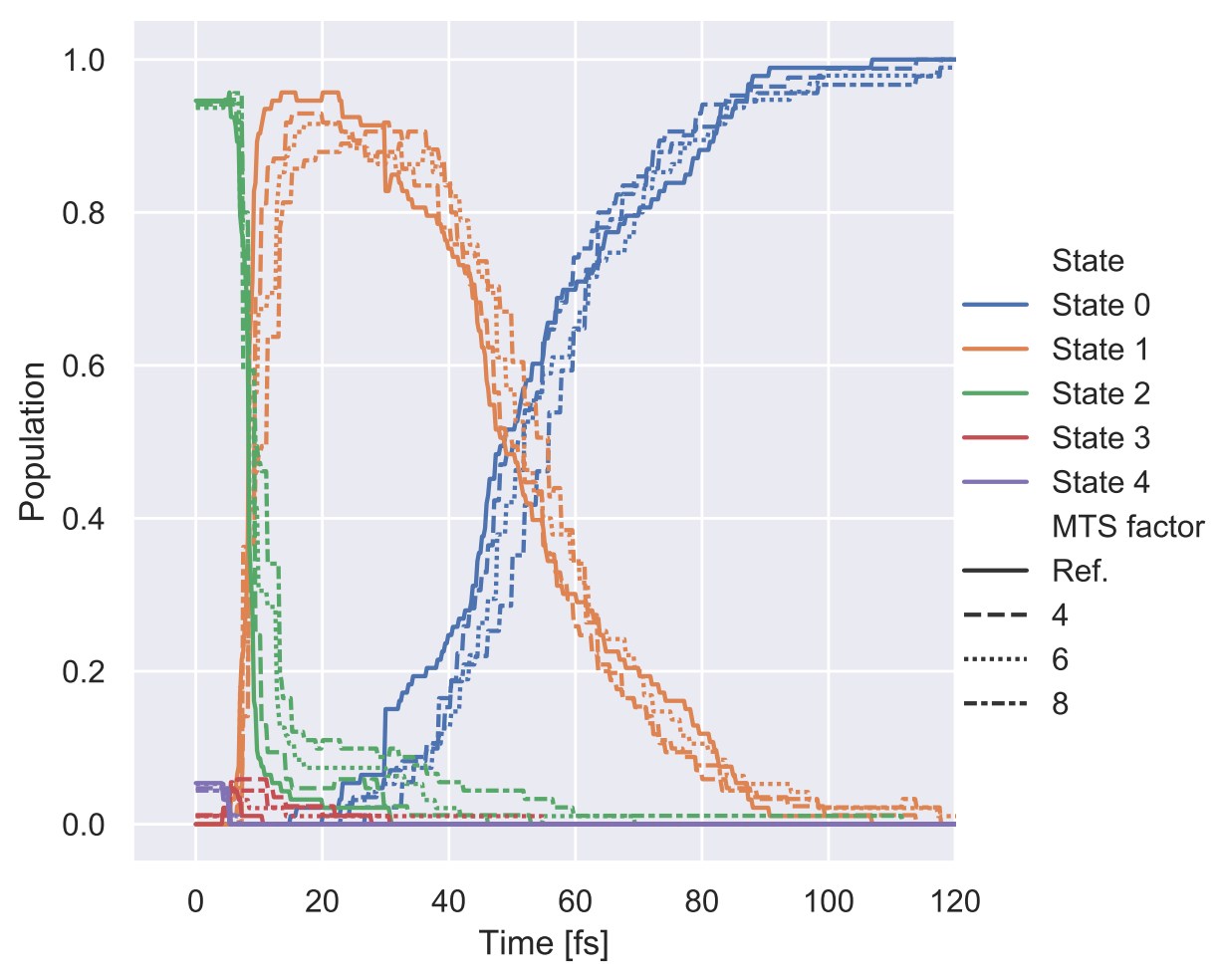

FIG. 6: Collective evolution of the state populations along the FS-TSH/PBE0 and MTS-TSH molecular dynamics. For the MTS-TSH runs, the PBE0 functional was used as high level, while the PBE functional was used for the low level forces.

With those initial conditions, 4 different types of simulations have been produced. A reference FS-TSH/PBE0 set of runs, and 3 different MTS-TSH batches with $N=\{4,6,8\}$ and using the PBE0 functional as high level and the PBE functional as low level. In total 400 trajectories were thus obtained. Each simulation is stopped when a transition to the ground state occurs or when it reaches a region of the PES where the calculation fails to converge. Most simulations reach the ground state in less than 100 fs. The calculations that failed to converge have not been considered in the statistical analysis below. In the reference calculations, 7 failed to converge, while for the MTS runs 15, 5, and 9 failed to converge for the MTS factor 4, 6, and 8, respectively. This seems to indicate that the MTS algorithm does not affect the convergence of the calculations significantly.

In Fig. 6, the collective evolution of the state populations along the dynamics are represented. The main characteristics of the photorelaxation process are well reproduced by all three MTS-TSH runs. In particular, the population is transferred from $S_{2}$ to $S_{1}$ in the 
first 10 to 20 fs and then slowly decays into the ground state. We note that the tail of the population of $S_{2}$ seem to become larger with the MTS factor, which shows the limit of the MTS scheme. This is reflected in the average lifetime of $S_{2}$, for which we get 8.7 fs in the reference runs, while the MTS simulations lead to lifetimes of 10.1, 11.3, and 13.8 fs, for the MTS factors, 4, 6, and 8, respectively. The average lifetime of the first excited state is however well described with all simulations. The reference lifetime for $S_{1}$ is of $43.5 \mathrm{fs}$, while the MTS simulations lead to lifetimes of 42.5, 44.3, and 42.6 fs, for the MTS factors, 4, 6, and 8 , respectively.

The populations of $S_{3}$ and $S_{4}$ are negligible. However, the trajectories starting in $S_{4}$ shows that different decay mechanisms are possible, hopping directly from $S_{4}$ to $S_{2}$ and then to $S_{1}$ or hopping first to $S_{3}$ and then directly to $S_{1}$. Those mechanisms are rare due to the fact that the initial population of $S_{4}$ is much lower than the population of $S_{2}$, but they are also part of the MTS-TSH swarm of trajectories, which indicates that the new MTS approach is reliable.

Following the work of Westermayr et al. in Ref. 9, we have analyzed the geometries at which the $S_{2}$ to $S_{1}$ and $S_{1}$ to $S_{0}$ transitions occur. Fig. 7 represents the values of relevant geometrical parameters at the hopping geometries for the first and second transitions, respectively. From Fig. 7, we can see that the hopping geometries from the MTS-TSH runs spread basically over the same region as the hopping geometries from the reference TSH simulations.

As the MTS factor is increased, the average number of standard high level steps per trajectory will decrease, which should decrease the computational cost of the MTS-TSH method. By standard high level steps, it is meant, high level steps which are not triggered by a low-level (LZ) transition. The number of triggered high level steps should increase with the MTS factor, since the average number of isolated low level steps (not linked to a high level calculation) will increase. Indeed, we obtain an average number of standard high level steps per trajectory of 55.6, 39.2, and 29.7 for the MTS factors 4, 6, and 8, respectively, while the average number of triggered high level steps per trajectory is 2.0, 2.5, and 2.6 for the MTS factors 4, 6, and 8 respectively.

Regarding the computational efficiency, the real speed-ups obtained over all the trajectories are 2.08, 2.56 and 2.86 for the MTS factors 4, 6, and 8, respectively. These speed-ups are in good agreement with the ideal speed-ups reporter in Fig. 5, which are 2.24, 2.76, and 


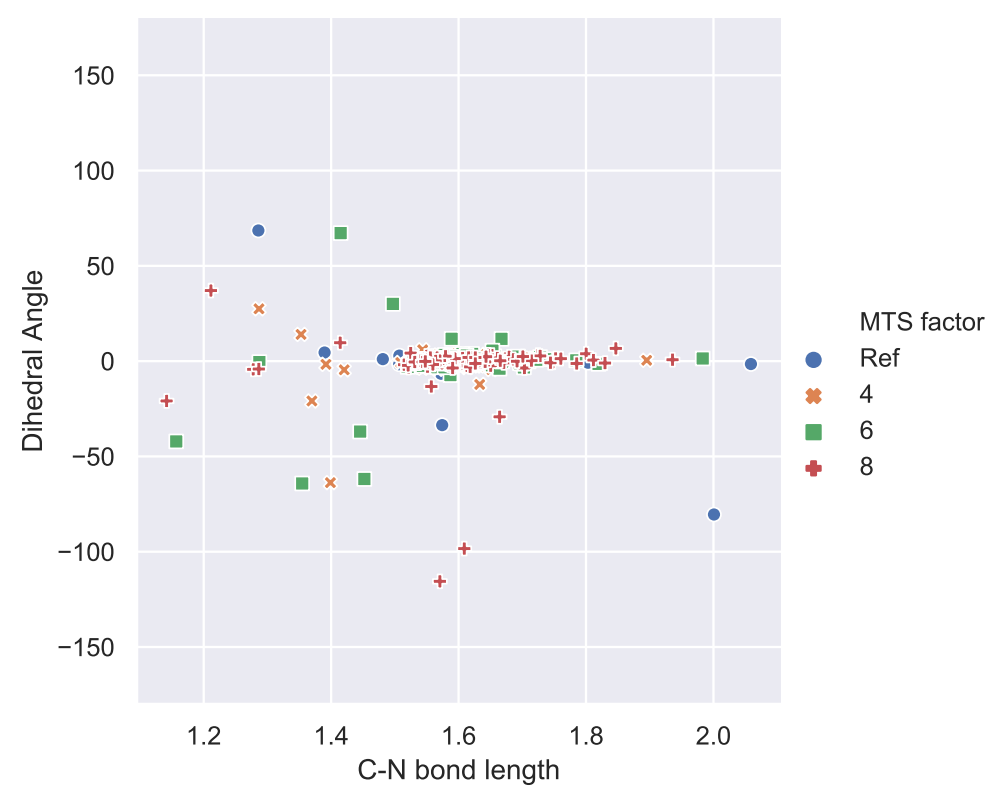

(a) Geometry analysis of electronic transitions from $\mathrm{S}_{2}$ to $\mathrm{S}_{1}$.
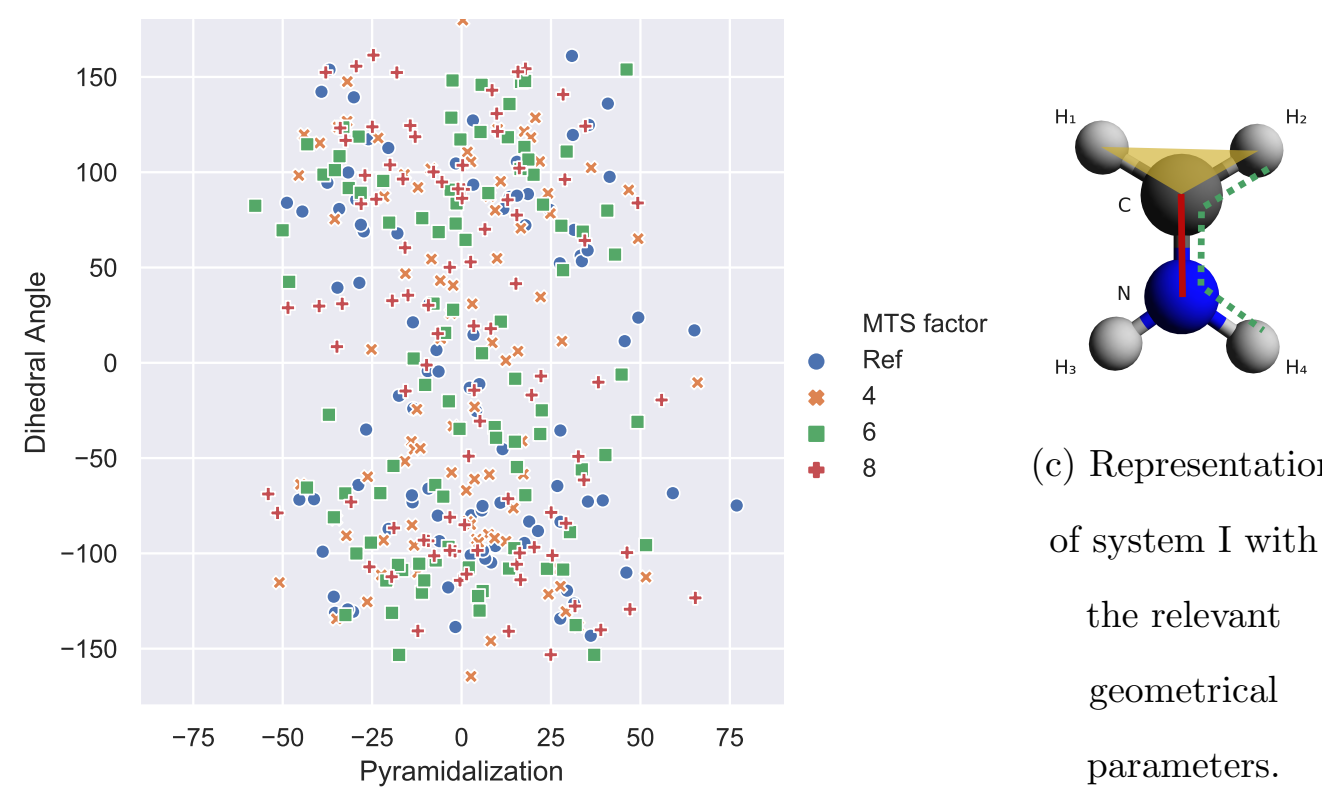

(c) Representation of system I with the relevant geometrical parameters.

(b) Geometry analysis of electronic transitions from $\mathrm{S}_{1}$ to $\mathrm{S}_{0}$.

FIG. 7: Representations of the hopping geometries for the $S_{2}$ to $S_{1}$ transition in Fig. 7a and for the $\mathrm{S}_{1}$ to $\mathrm{S}_{0}$ transition in Fig. 7b. In Fig. 7c, the C-N bond length is represented with the red solid line, the dihedral Angle between atoms $\left(\mathrm{H}_{4}, \mathrm{~N}, \mathrm{C}\right.$, and $\left.\mathrm{H}_{2}\right)$ is represented with the green dotted line, while the pyramidalization angle corresponds to the angle between the red solid line and the yellow triangle. 


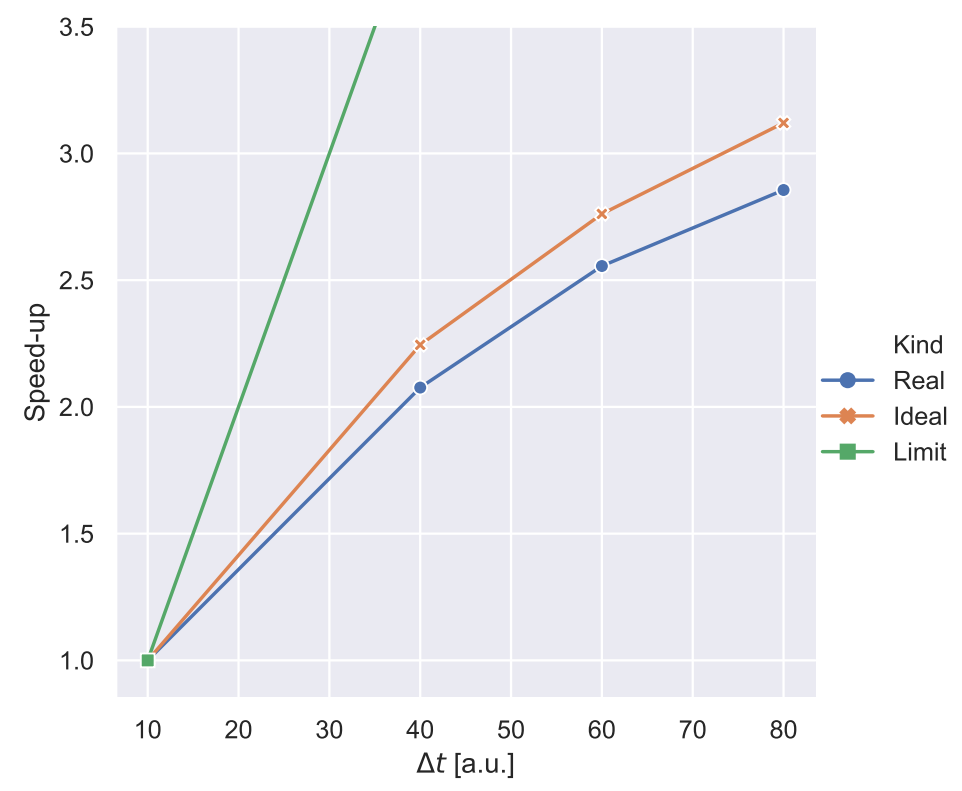

FIG. 8: Real MTS speed-ups obtained from MTS-TSH calculations with different MTS factors compared to reference PBE0 FS-TSH timings. For comparison the ideal and limit speed-ups as defined in eqs. (21) and (22), respectively, are also reported. The ideal speed-ups are identical to the ones in Fig. 5.

3.12 for the MTS factors 4, 6, and 8, respectively, as can be seen from Fig. 8.

Overall, this statistical investigation indicates that the MTS-TSH algorithm introduced in section II B allows to reproduce results from standard TSH simulations with a significant speed-up.

\section{CONCLUSIONS AND OUTLOOK}

We have presented a new algorithm for non-adiabatic molecular dynamics simulations that is based on Tully's FS-TSH method combined with an MTS scheme for the integration of the nuclear classical equations of motion. The MTS scheme is an extension of the CPMD implementation introduced by Liberatore et al. in Ref. 21, in which the decomposition of the forces in terms of slow and fast components relies on the use of different electronic structure methods (e.g. different DFT functionals). In order to adapt the MTS scheme to the TSH method, it is important to enable electronic transitions in between outer steps. This is achieved by pre-evaluating the transition probabilities during inner steps with a low- 
level LZ criterion. If a transition is detected, a high level calculation is triggered, to confirm (or not) the electronic transition.

This new MTS-TSH algorithm has been tested successfully on the photorelaxation of protonated formaldimine. We have shown that the MTS-TSH method is able to recover the correct state population along the reaction path as well as the correct geometries at the transitions. For this MTS scheme (combining PBE/PBE0 forces and time step factors between 2 and 8) a speed-up between 1.5 and 3 could be achieved compared to standard FS-TSH simulations. The obtained speed-ups are actually very close to the ideal speed-up that could be obtained with the computational settings considered, indicating that a better performance could be reached by considering cheaper models as low level in the MTS scheme.

This work constitutes a preliminary investigation and more tests should be performed on more complex systems to confirm the reliability of the presented results. Nonetheless, the presented MTS-TSH algorithm has shown promising results and this formulation opens the door to new developments such as combinations with other electronic structure models including machine learning techniques and QM/MM frameworks to target larger molecular systems and obtain computationally even less demanding algorithms for the description of non-adiabatic phenomena.

\section{SUPPLEMENTARY MATERIAL}

All the computational details and raw data from section III are provided at https: //doi.org/10.5281/zenodo.3459170, including CPMD input and output files as well as analysis scripts.

\section{AUTHOR INFORMATION}

\section{Contributions}

Conceptualization: P.B. (supporting), and U.R. (lead); Methodology: P.B. (lead) and U.R. (supporting); Software: P.B.; Investigation: P.B. (lead) and U.R. (supporting); Resources: U.R.; Writing - Original Draft: P.B.; Writing - Review and Editing: P.B (lead), and U.R. (supporting); Supervision: U.R.; Funding Acquisition: U.R. 


\section{Funding}

U.R. acknowledges funding from the Swiss National Science Foundation via the NCCR MUST and individual grants. The calculations in section III C have been performed using the Intel Broadwell based FIDIS cluster facilities of the Scientific IT and Application Support Center of EPFL.

\section{Notes}

The authors declare no competing financial interest.

\section{ACKNOWLEDGMENTS}

P.B. thanks Martin P. Bircher for his help with the CPMD package and Elisa Liberatore for valuable discussions.

\section{REFERENCES}

${ }^{1}$ B. F. E. Curchod and T. J. Martínez, Chem. Rev. , acs.chemrev.7b00423 (2018).

${ }^{2}$ R. Crespo-otero and M. Barbatti, Chem. Rev. 118, 7026 (2018).

${ }^{3}$ T. Nelson, S. Fernandez-Alberti, V. Chernyak, A. E. Roitberg, and S. Tretiak, J. Chem. Phys. 136, 054108 (2012).

${ }^{4}$ M. Barbatti, Wiley Interdiscip. Rev. Comput. Mol. Sci. 1, 620 (2011).

${ }^{5}$ T. Helgaker, P. Jørgensen, and J. Olsen, Molecular Electronic-Structure Theory, 1st ed. (John Wiley \& Sons, Ltd, Chichester, UK, 2000).

${ }^{6}$ F. Jensen, Introduction to Computational Chemistry, 2nd ed. (John Wiley \& Sons, Ltd, Chichester, UK, 2007).

${ }^{7}$ L. Wang, A. Akimov, and O. V. Prezhdo, J. Phys. Chem. Lett. 7, 2100 (2016).

${ }^{8}$ P. O. Dral, M. Barbatti, and W. Thiel, J. Phys. Chem. Lett. 9, 5660 (2018).

${ }^{9}$ J. Westermayr, M. Gastegger, M. F. S. J. Menger, S. Mai, L. González, and P. Marquetand, Chem. Sci. (2019), 10.1039/C9SC01742A, arXiv:1811.09112.

${ }^{10}$ M. E. Tuckerman, B. J. Berne, and G. J. Martyna, J. Chem. Phys. 97, 1990 (1992). 
11 "CPMD, http://www.cpmd.org/, Copyright IBM Corp 1990-2015, Copyright MPI für Festkörperforschung Stuttgart 1997-2001." .

${ }^{12}$ J. C. Tully and R. K. Pkeston, J. Chem. Phys. 55, 562 (1971).

${ }^{13}$ J. C. Tully, J. Chem. Phys. 93, 1061 (1990).

${ }^{14}$ M. Barbatti and R. Crespo-Otero, in Density-Functional Methods Excit. States, edited by N. Ferré, M. Filatov, and M. Huix-Rotllant (Springer, Cham, 2015) pp. 415-44.

${ }^{15}$ E. Tapavicza, I. Tavernelli, and U. Rothlisberger, Phys. Rev. Lett. 98, 1 (2007).

${ }^{16}$ M. E. Casida, in Recent Adv. Density Funct. Methods, Part I, edited by D. P. Chong (World Scientific, Singapore, 1995) p. 155.

${ }^{17}$ S. Hammes-Schiffer and J. C. Tully, J. Chem. Phys. 101, 4657 (1994).

${ }^{18}$ G. A. Jones, B. K. Carpenter, and M. N. Paddon-Row, J. Am. Chem. Soc. 120, 5499 (1998).

${ }^{19}$ H. Nakamura, Nonadiabatic Transition (World Scientific, Singapore, 2002).

${ }^{20}$ I. Tavernelli, E. Tapavicza, and U. Rothlisberger, J. Chem. Phys. 130, 124107 (2009).

${ }^{21}$ E. Liberatore, R. Meli, and U. Rothlisberger, J. Chem. Theory Comput. 14, 2834 (2018).

${ }^{22}$ H. F. Trotter, Proc. Am. Math. Soc. 10, 545 (1959).

${ }^{23}$ R. P. Steele, J. Chem. Phys. 139, 011102 (2013).

${ }^{24}$ M. Barbatti, A. J. A. Aquino, and H. Lischka, Mol. Phys. 104, 1053 (2006).

${ }^{25}$ I. Tavernelli, E. Tapavicza, and U. Rothlisberger, J. Mol. Struct. THEOCHEM 914, 22 (2009).

${ }^{26}$ S. A. Fischer, B. F. Habenicht, A. B. Madrid, W. R. Duncan, and O. V. Prezhdo, J. Chem. Phys. 134, 024102 (2011).

${ }^{27}$ J. P. Perdew, M. Ernzerhof, and K. Burke, J. Chem. Phys. 105, 9982 (1996).

${ }^{28}$ J. P. Perdew, K. Burke, and M. Ernzerhof, Phys. Rev. Lett. 77, 3865 (1996).

${ }^{29}$ J. P. Perdew, K. Burke, and M. Ernzerhof, Phys. Rev. Lett. 78, 1396 (1997).

${ }^{30}$ P. Minary, M. E. Tuckerman, and G. J. Martyna, Phys. Rev. Lett. 93, 150201 (2004). 